\title{
ARTICLE
}

Received 18 Oct 2010 | Accepted 9 Mar 2011 | Published 5 Apr 2011

DOI: $10.1038 /$ ncomms1269

\section{Neural crest cells organize the eye via TGF- $\beta$ and canonical Wnt signalling}

Timothy Grocott', Samuel Johnson'1, Andrew P. Bailey ${ }^{1, i} \&$ Andrea Streit ${ }^{1}$

In vertebrates, the lens and retina arise from different embryonic tissues raising the question of how they are aligned to form a functional eye. Neural crest cells are crucial for this process: in their absence, ectopic lenses develop far from the retina. Here we show, using the chick as a model system, that neural crest-derived transforming growth factor- $\beta$ s activate both Smad3 and canonical Wnt signalling in the adjacent ectoderm to position the lens next to the retina. They do so by controlling Pax6 activity: although Smad3 may inhibit Pax6 protein function, its sustained downregulation requires transcriptional repression by Wnt-initiated $\beta$-catenin. We propose that the same neural crest-dependent signalling mechanism is used repeatedly to integrate different components of the eye and suggest a general role for the neural crest in coordinating central and peripheral parts of the sensory nervous system. 
$\mathrm{n}$ the vertebrate head, different components of the sensory nervous system develop from different embryonic tissues. For example, in the eye, the retina arises from the central nervous system, whereas the lens originates in the surface ectoderm ${ }^{1}$. An unresolved problem is how these components are aligned with each other to ensure normal structure and function. In the eye, it is generally accepted that the lens is induced by the optic vesicle (future retina) ${ }^{1-3}$, which would explain their alignment. However, we now know that lens induction begins before contact with the optic vesicle, and that much of the cranial ectoderm has intrinsic lens potential ${ }^{4-6}$. Therefore, lens potential must be actively suppressed in non-lens ectoderm. Our previous observations implicate neural crest cells (NCCs) in this process: NCC ablation in vivo causes ectopic lenses to develop, and NCCs repress lens specification in vitro ${ }^{4,6}$. Consistent with this, lens potential becomes gradually restricted during NCC migration ${ }^{4,5,7}$ and NCCs invade most areas of the head, but never reach the future lens because the optic vesicle functions as a physical barrier ${ }^{8-9}$. Thus, NCCs inhibit lens development except in the vicinity of the retina, ensuring the alignment of peripheral (lens) and central (retina) components of the eye. However, the signals involved are unknown.

Here we show that NCC-derived members of the transforming growth factor (TGF)- $\beta$ subfamily activate both Smad 3 and canonical Wnt signalling in the non-lens ectoderm. In the lens territory, both pathways prevent lens formation; however, their inhibition prevents lens suppression by NCCs. Thus, Smad3-mediated TGF- $\beta$ and canonical Wnt signalling cooperate to restrict lens potential to the ectoderm overlying the retina, and thus have a critical role in the formation of a functional eye.

\section{Results}

TGF- $\boldsymbol{\beta}$ signalling inhibits lens specification. The transcription factor Pax6 has a key role in eye development and is required for lens formation; it activates its own transcription as well as that of other lens-specific genes ${ }^{10-14}$. However, in vitro its autoregulation can be inhibited at the protein level by binding to phosphorylated Smad3 (pSmad3) after TGF- $\beta$ stimulation ${ }^{15}$, implicating this as a candidate pathway for lens restriction. We found that multiple TGF- $\beta$ ligands are expressed in migrating NCCs, but are absent from the lens, these included TGF- $\beta 1$, Activin- $\beta A$ and Activin- $\beta B$ (Supplementary Fig. S1). Following contact with migrating NCCs, the adjacent non-lens ectoderm received TGF- $\beta$ signals as shown by the presence of $\mathrm{pSmad} 3$, whereas lens ectoderm, which was protected from infiltrating NCCs by the optic vesicle, was pSmad3-negative (Supplementary Fig. S2). Thus, members of the TGF- $\beta$ subfamily, which signal through Smad2 and Smad3 (hereafter referred to as TGF- $\beta$ for simplicity), are expressed in NCCs, and only non-lens ectoderm activates this pathway.

To assess whether activated Smad3 suppresses lens fate in vivo, we electroporated constitutively active Smad3 (ref. 16) into the presumptive lens ectoderm (PLE). Although control green fluorescent protein-expressing cells were incorporated into the lens and express the lens marker $\delta$-crystallin (Fig. 1a-c; $3 / 3$ lenses), constitutively active Smad3-expressing cells were excluded from the lens and did not express $\delta$-crystallin (Fig. 1d-f; 0/5 lenses). Together, the above results suggest that TGF- $\beta$ s prevent lens formation at inappropriate positions.

To study this further, we used an explant system that recapitulates endogenous lens development: pre-placodal gene expression (Six1, Eya2; Fig. 1g,h; see Table 1 for numbers of all explant experiments) preceded the expression of Pax6, L-Maf and $\delta$-crystallin (Fig. 1i-k), each of which labels a distinct phase of lens formation. This allowed us to determine the stage at which TGF- $\beta / \mathrm{Smad} 3$ function to inhibit lens development. Culture of explants in Activin A did not affect the expression of early pre-placodal genes (Fig. 11,m), but did prevent the expression of the later lens-specific markers (Fig. 1n-p). In cells
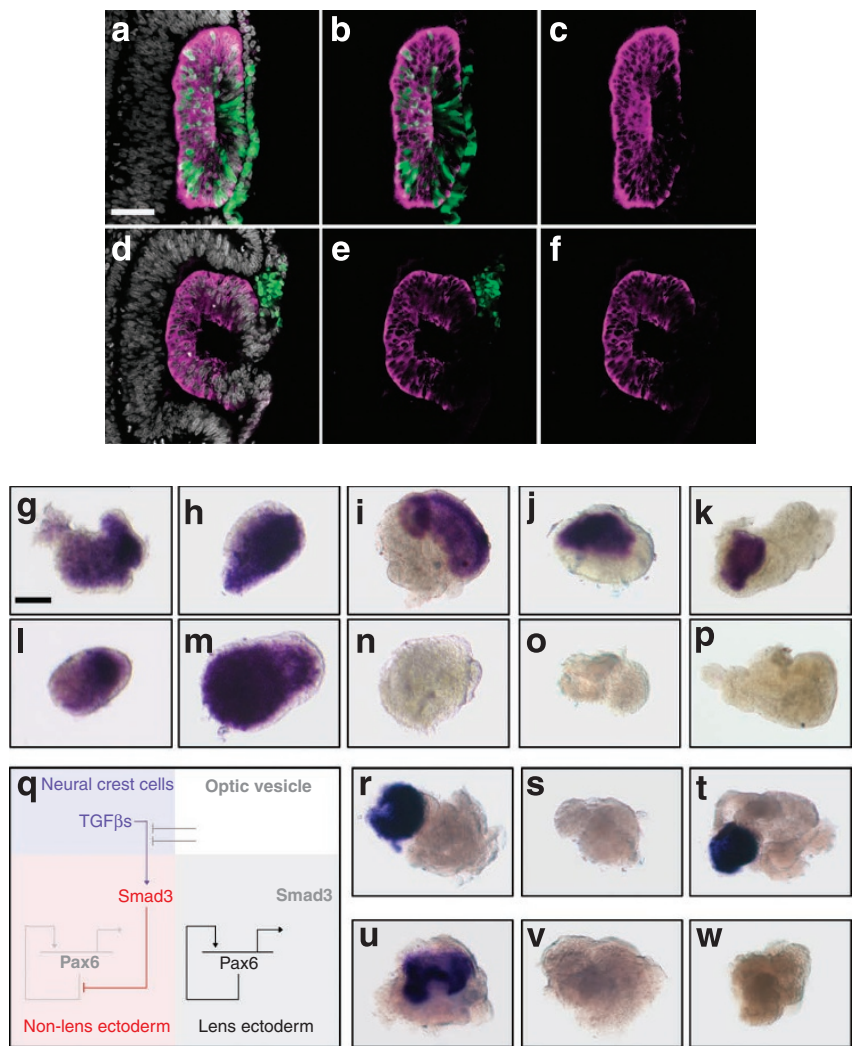

Figure 1 | TGF- $\boldsymbol{\beta}$ represses lens formation. (a-c) Presumptive lens ectoderm (PLE) was electroporated with expression vector encoding green fluorescent protein (GFP): (a) GFP-expressing cells (green) counterstained for $\delta$-crystallin (magenta) and cell nuclei (grey); (b) GFP and $\delta$-crystallin staining only; (c) $\delta$-crystallin staining only. (d-f) PLE was electroporated with expression vector encoding constitutively active Smad3 + GFP:

(d) GFP-expressing cells (green) counterstained for $\delta$-crystallin (magenta) and cell nuclei (grey); (e) GFP and $\delta$-crystallin staining only; (f) $\delta$-crystallin staining only. (g-k) PLE explants were cultured in vitro and assayed for pre-placodal (Six1, Eya2) or lens (Pax6, L-Maf, $\delta$-crystallin) gene expression: (g) Six1; (h) Eya2; (i) Pax6; (j) L-Maf; and (k) $\delta$-crystallin. (I-p) PLE explants were cultured in the presence of Activin $A$ and assayed for pre-placodal or lens gene expression: (I) Six1; (m) Eya2; (n) Pax6; (o) L-Maf; and (p) $\delta$-crystallin. (q) Model for lens inhibition by TGF- $\beta$ signalling. (r-t) PLE explants were cultured alone or in combination with Activin A or SB431542 and assayed for $\delta$-crystallin gene expression: (r) PLE alone; (s) PLE + Activin A; and (t) PLE + Activin A + SB431542. (u-w) PLE explants were cultured alone or in combination with neural crest cells (NCC) or SB431542 and assayed for $\delta$-crystallin gene expression: (u) PLE alone; (v) PLE +NCC; and (w) PLE + NCC + SB431542. Scale bars: $\mathbf{a}, 50 \mu \mathrm{m}$ for panels $\mathbf{a}-\mathbf{f}, \mathbf{g}, 50 \mu \mathrm{m}$ for panels $\mathbf{g}-\mathbf{p}, \mathbf{r}-\mathbf{w}$

in which lens markers were repressed, markers for other sensory structures were not induced (Table 1; $\mathrm{Pax}^{+}$trigeminal, $\mathrm{Pax2}^{+}$otic or Fox $\mathrm{F}^{+}$olfactory placodes), suggesting that Activin A maintains pre-placodal cells in an undifferentiated progenitor state, as shown for other cell types ${ }^{17,18}$. Thus, activation of TGF- $\beta$ signalling prevents the acquisition of lens fate in vivo and in vitro and is likely to function by regulating Pax6. We propose that NCC-derived TGF$\beta$ s activate Smad3 in the overlying surface ectoderm, which may in turn sequester Pax6 protein ${ }^{15}$ to prevent activation of downstream targets and lens formation (Fig. 1q).

Wnt signalling is required for lens repression by NCCs. If TGF- $\beta$ signalling alone is responsible for positioning the lens, inhibition 
Table 1 | Summary of in vitro experiments.

\begin{tabular}{|c|c|c|c|c|c|c|c|c|c|}
\hline \multirow{2}{*}{$\begin{array}{l}\text { Explanted tissues } \\
\text { and experimental } \\
\text { conditions }\end{array}$} & \multicolumn{9}{|c|}{ Marker genes } \\
\hline & Six1 & Eya2 & FoxG1 & Pax2 & Pax3 & Pax6 & L-Maf & $\delta$-Crystallin & Wnt2b \\
\hline PLE & $100 \%(7 / 7)$ & $90 \%(19 / 21)$ & $0 \%(0 / 13)$ & $0 \%(0 / 12)$ & $0 \%(0 / 12)$ & $93 \%(81 / 87)$ & $83 \%(10 / 12)$ & $80 \%(76 / 95)$ & $0 \%(0 / 9)$ \\
\hline PLE + Activin & $92 \%(12 / 13)$ & $100 \%(8 / 8)$ & $0 \%(0 / 12)$ & $0 \%(0 / 11)$ & $0 \%(0 / 13)$ & $18 \%(9 / 50)$ & $18 \%(2 / 11)$ & $13 \%(1 / 8)$ & $90 \%(9 / 10)$ \\
\hline $\mathrm{PLE}+$ Activin + N-Fz8 & - & - & - & - & - & $83 \%(15 / 18)$ & - & - & - \\
\hline $\mathrm{PLE}+\mathrm{NCC}$ & - & - & - & - & - & $60 \%(9 / 15)$ & - & $9 \%(2 / 23)$ & - \\
\hline $\mathrm{PLE}+\mathrm{NCC}+\mathrm{N}-\mathrm{Fz} 8$ & - & - & - & - & - & - & - & $60 \%(3 / 5)$ & - \\
\hline PLE + DMSO & - & - & - & - & - & - & - & $95 \%(18 / 19)$ & $11 \%(2 / 19)$ \\
\hline PLE + Activin + DMSO & - & - & - & - & - & - & - & $20 \%(2 / 10)$ & - \\
\hline PLE + Activin + SB431542 & - & - & - & - & - & - & - & $74 \%(14 / 19)$ & - \\
\hline $\mathrm{PLE}+\mathrm{NCC}+\mathrm{DMSO}$ & - & - & - & - & - & - & - & $14 \%(5 / 35)$ & $44 \%(7 / 16)$ \\
\hline $\mathrm{PLE}+\mathrm{NCC}+\mathrm{SB} 431542$ & - & - & - & - & - & - & - & $14 \%(3 / 21)$ & $17 \%(1 / 6)$ \\
\hline
\end{tabular}

of this pathway should rescue lens development in the presence of NCCs. To test this, we made use of the TGF- $\beta$ receptor inhibitor SB431542 (SB $)^{19}$. When cultured with both Activin A and SB, presumptive lens explants retained $\delta$-crystallin expression, in contrast to treatment with Activin A alone (Fig. 1r-t). However, SB was unable to restore lens marker expression in the presence of presumptive NCCs (Fig. $1 \mathrm{u}-\mathrm{w}$ ), suggesting that, although TGF- $\beta$ signalling is sufficient for lens inhibition, other signals must be involved.

In mouse, canonical Wnt signalling suppresses lens development ${ }^{20}$. Consistent with this, we found that overexpression of constitutively active $\beta$-catenin in PLE prevented the expression of $\delta$-crystallin. Although $83 \%$ of control cells were $\delta$-crystallin ${ }^{+}(66 / 87$ cells in three lenses; Fig. $2 \mathrm{a}-\mathrm{c}$ ), this was reduced to $35 \%$ when canonical Wnt signalling was activated (27/77 cells in five lenses; Fig. $2 d-j$ ). Interestingly, similar to caSmad3-expressing cells (Fig. 1d-f), those carrying $\beta$-catenin were excluded from the lens over time: after $24 \mathrm{~h}$ $\beta$-catenin ${ }^{+} / \delta$-crystallin ${ }^{-}$cells were found in the lens placode (Fig. $2 \mathrm{~d}-\mathrm{f}$ ), but were mostly absent from the lens after $48 \mathrm{~h}$ (Fig. $2 \mathrm{~g}-\mathrm{i}$ ). If Wnt signalling is involved in the residual lens-repressing activity of NCCs, Wnt inhibition should rescue lens development in the presence of NCCs. To test this, we cocultured NCCs and PLE with the Wnt antagonist N-Fz8. Indeed, inhibition of Wnt signalling rescued $\delta$-crystallin expression (Fig. $2 \mathrm{k}-\mathrm{m}$ ), suggesting that Wnt is required for lens repression by NCCs and suppresses lens formation through $\beta$-catenin (Fig. 2n).

Wnt represses lens fate downstream of NCC-derived TGF- $\beta$ s. In a survey of Wnt expression, we did not find any Wnt transcripts present in migrating NCCs. However, we did find a strong correlation between NCC migration and Wnt2b expression in the ectoderm: following contact with migrating NCCs, $W n t 2 b$ began to be expressed in the neighbouring ectoderm but remained absent from the prospective lens territory (Fig. 3a-c). The highest levels of Axin2 expression (a read-out of canonical Wnt activity) were present in ectoderm that had been in contact with NCCs for longest duration (Fig. 3d,e, arrowheads; compare midbrain and forebrain levels). These observations suggest that NCCs initiate $W n t 2 b$ expression in the ectoderm in which lens formation is normally inhibited. Indeed, migratory NCCs strongly induced $W n t 2 b$ in presumptive lens explants, which normally do not express the transcript (Fig. 3f,g), suggesting that TGF- $\beta$ may be the NCC signal responsible for ectodermal $W n t 2 b$. Indeed, in vitro, Wnt $2 b$ induction by NCCs failed when TGF- $\beta$ signalling was inhibited (Fig. 3h), whereas Activin A strongly induced $W n t 2 b$ in presumptive lens explants (Fig. 3i,j). Similarly, in vivo, ectodermal Wnt $2 b$ expression depended on TGF- $\beta$ signalling from NCCs: NCC ablation (Fig. $3 \mathrm{k}, 1$ ) and misexpression of the inhibitory Smad7 (Fig. 3m,n; Smad7: 2/6 Wnt2b+,
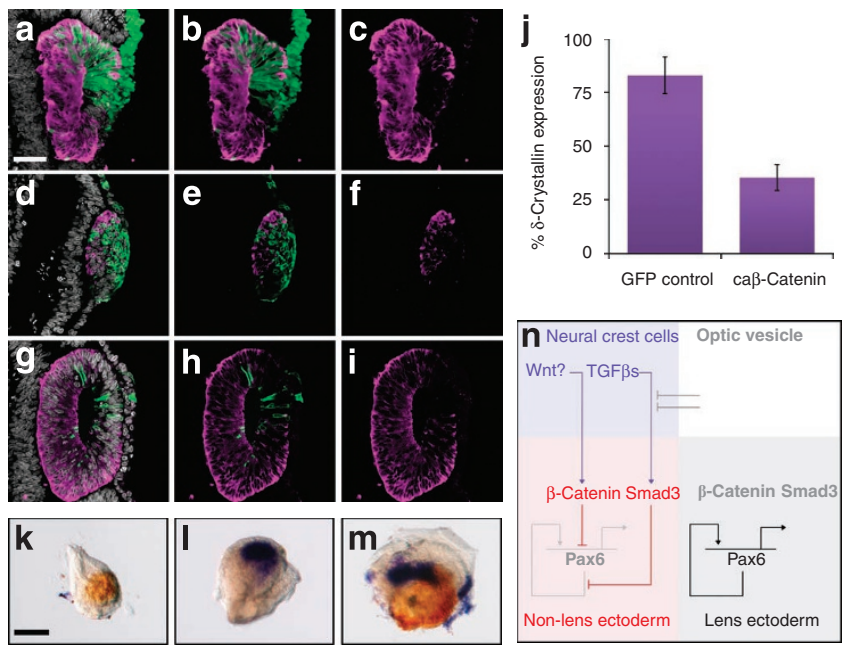

$\mathbf{K}$

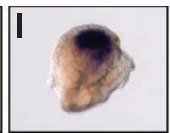

Non-lens ectoderm

Lens ectoderm

Figure 2 | $\beta$-Catenin inhibits lens formation, and Wnt signalling is required for lens repression by NCCs. (a-c). Presumptive lens ectoderm (PLE) was electroporated with expression vector encoding green fluorescent protein (GFP) and embryos were cultured for $48 \mathrm{~h}$. (a) GFPexpressing cells (green) were stained for $\delta$-crystallin protein (magenta) and cell nuclei (grey); (b) GFP and $\delta$-crystallin staining only; (c) $\delta$-crystallin staining only. (d-i) PLE was electroporated with caß-catenin + GFP and embryos were cultured for 24 or $48 \mathrm{~h}$. (d-f) Twenty-four hours: (d) GFPexpressing cells (green) were stained for $\delta$-crystallin protein (magenta) and cell nuclei (grey); (e) GFP and $\delta$-crystallin staining only; (f) $\delta$-crystallin staining only. (g-i) Forty-eight hours: (g) GFP-expressing cells (green) were stained for $\delta$-crystallin protein (magenta) and cell nuclei (grey); (h) GFP and $\delta$-crystallin staining only; (i) $\delta$-crystallin staining only. (j) Mean proportion of $\mathrm{GFP}^{+}$electroporated cells within the lens that also express $\delta$-crystallin protein. GFP control: $n=3$ lenses; 66 of 87 electroporated cells express $\delta$-crystallin. caß-catenin: $n=5$ lenses; 27 of 77 electroporated cells $\delta$-crystallin. Error bars: \pm 1 s.d. (k-m) PLE explants were cultured alone or in combination with neural crest cells (NCCs) or N-Fz8 and assayed for lens ( $\delta$-crystallin; brown) and NCC (CRABP1; blue) gene expression: (k) PLE alone; (I) PLE + NCC; (m) PLE + NCC + N-Fz8. (n) Model for lens inhibition by TGF- $\beta$ and canonical Wnt signalling. Scale bars: $\mathbf{a}, 50 \mu \mathrm{m}$ for panels a-i; k, $50 \mu \mathrm{m}$ for panels $\mathbf{k}-\mathbf{m}$.

green fluorescent protein: 5/5 Wnt $2 b^{+}$) caused downregulation of $W n t 2 b$ transcripts in the non-lens ectoderm. Together, these results show that TGF- $\beta$ signalling from NCCs induces $W n t 2 b$ expression in the non-lens ectoderm. 

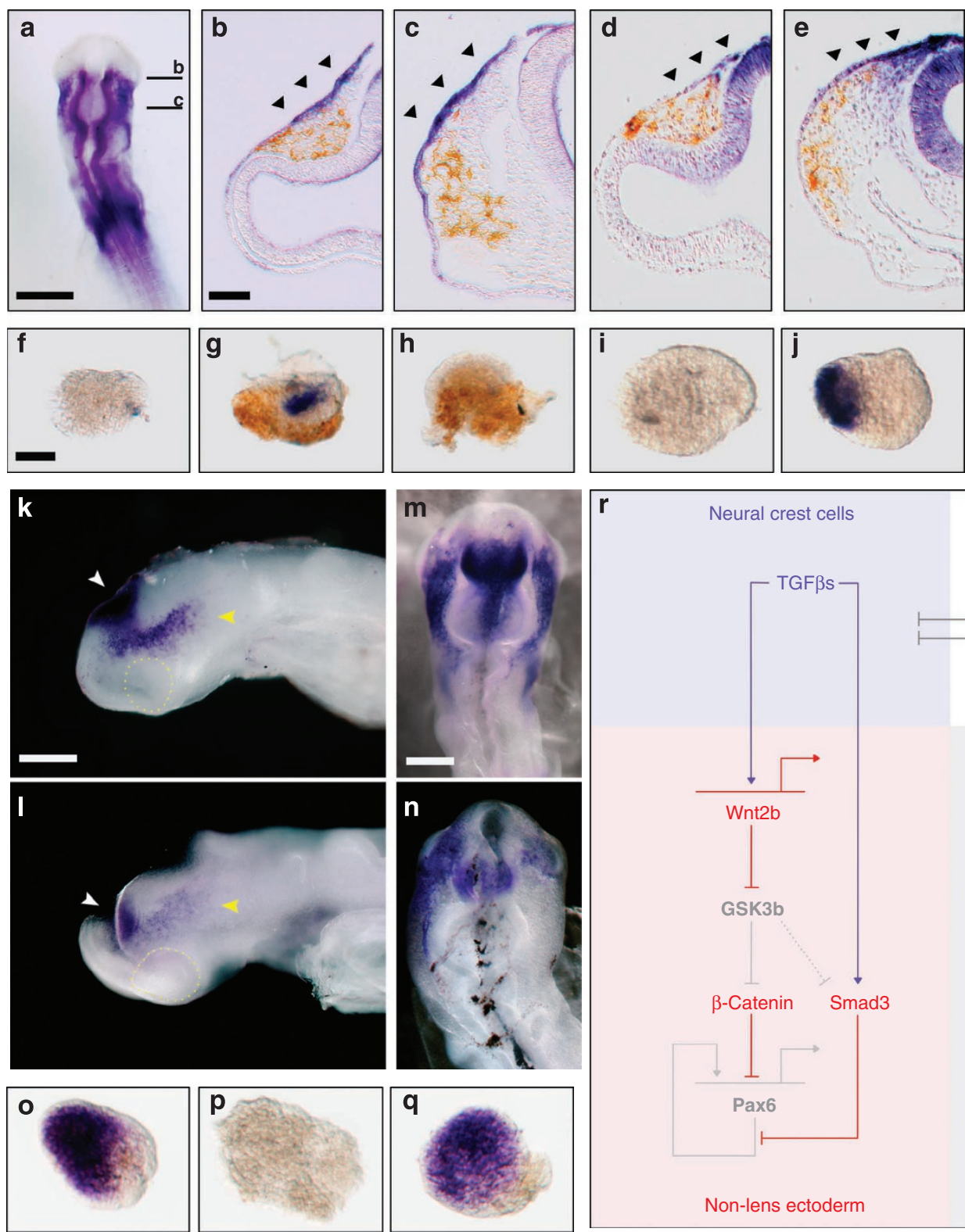

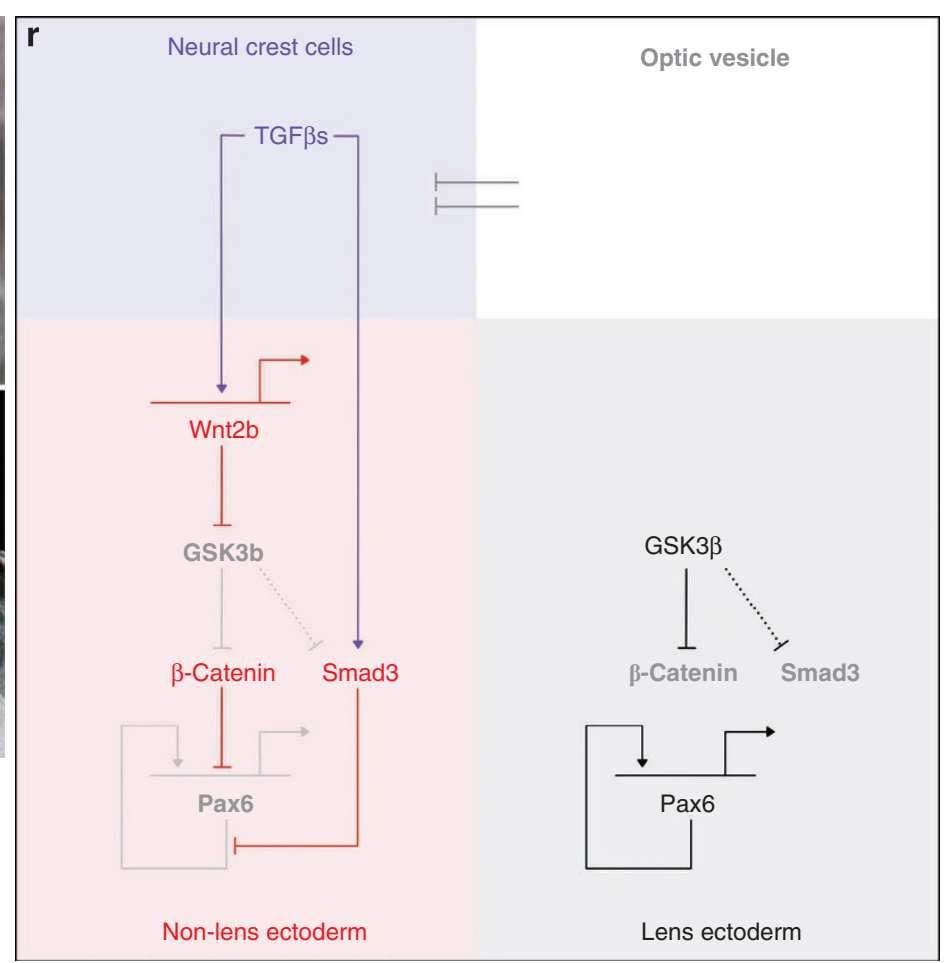

Figure $\mathbf{3}$ | Wnt mediates lens repression by NCCs and TGF- $\boldsymbol{\beta}$. (a-c) Wnt2b (blue) gene expression during neural crest cell (NCC) migration: (a) Wholemount embryo showing the forebrain section used in panel $\mathbf{b}$ and the midbrains section used in panel $\mathbf{c}$. (b) Forebrain level section stained for Wnt2b; (c) midbrain level section stained for Wnt2b. (d) Forebrain level section stained for Axin2 gene expression (blue). (e) Midbrain level section showing Axin2 expression. All sections are immunostained for NCC-specific HNK1 (brown). Arrowheads indicate gene expression in non-lens ectoderm. (f-h) Presumtive lens ectoderm (PLE) explants were cultured alone or in combination with NCC or SB431542 and assayed for Wnt2b gene expression (blue) and HNK1 (brown): (f) PLE alone; (g) PLE + NCC; (h) PLE + NCC + SB431542. (i, j) PLE explants were cultured alone or with Activin A and assayed for Wnt2b gene expression: (i) PLE alone; (j) PLE + Activin A. (k, I) Wnt2b gene expression (blue) following in ovo ablation of premigratory NCCs: (k) control embryo; (I) NCC ablated embryo. Compare Wnt2b expression in the brain (white arrowheads) with non-lens surface ectoderm (yellow arrowheads). (m, $\mathbf{n}$ ) Wnt $2 b$ gene expression (blue) following in ovo electroporation of expression vectors encoding green fluorescent protein (GFP) or Smad7 + GFP: (m) GFP; (n) Smad7 + GFP. (o-q) PLE explants were cultured alone or in combination with Activin A or N-Fz8 and assayed for Pax6 gene expression (blue): (o) PLE alone; (p) PLE + Activin A; (q) PLE + Activin A + N-Fz8. (r) Proposed molecular model to explain TGF- $\beta$ - and Wnt-mediated lens restriction. Broken lines: interactions inferred from the literature. (s) Proposed embryological model summarizing how NCCs organize the eye: NCCs (blue) secrete TGF- $\beta$ s, which signal to the non-lens ectoderm and dorsal optic vesicle. As a consequence, Wnt $2 b$ (red) is induced, and together they repress lens formation in the non-lens ectoderm. This leads to the alignment of Pax6 expression in the future lens and neural retina (grey). Scale bars: $\mathbf{a}, 500 \mu \mathrm{m} ; \mathbf{b}, 50 \mu \mathrm{m}$ for panels b-e; $\mathbf{f}, 50 \mu \mathrm{m}$ for panels $\mathbf{f}-\mathbf{j}, \mathbf{0}-\mathbf{q} ; \mathbf{k}, 250 \mu \mathrm{m}$ for panels $\mathbf{k}, \mathbf{l} ; \mathbf{m}, 250 \mu \mathrm{m}$ for panels $\mathbf{m}, \mathbf{n}$.

The above findings suggest that the lens-repressing activity of TGF- $\beta$ is mediated by Wnts. In the presence of Activin A, presumptive lens explants lose Pax6 transcripts (Figs 1i,n and 3o,p). However, when Wnt was simultaneously inhibited by N-Fz8, Pax6 expression was restored (Fig. 3q) demonstrating that lens suppression by TGF- $\beta$ depends on active Wnt signalling. Together, these findings support a model in which NCC-derived TGF- $\beta$ s initiate both $\operatorname{Smad} 3$ and canonical Wnt signalling to suppress lens fate in non-lens ectoderm (Fig. 3r,s).

\section{Discussion}

The formation of functional organs requires the integration of tissues with different embryonic origins; this is particularly evident 
in the vertebrate head in which peripheral components of sense organs or sensory ganglia must be perfectly aligned with their central counterparts to ensure normal function. Here we reveal the signalling network that controls this process in the vertebrate eye, in which the retina arises from the central nervous system and the lens from the surface ectoderm. Initial widespread lens potential ${ }^{4-7}$ is gradually restricted to the ectoderm next to the retina through complex interactions of TGF- $\beta$ and Wnt signalling (Fig. 3r,s). Migrating NCCs secrete TGF- $\beta$ s, which in turn activate Smad3 and canonical Wnt signalling in the adjacent non-lens ectoderm. Although our studies implicate Wnt $2 \mathrm{~b}$ as the source of Wnt activity, it is possible that other, yet to be identified, ligands have a role. We propose that, to position the lens, TGF- $\beta$ s initiate a 'double assurance' mechanism to regulate Pax6 function and transcription. Although pSmad3 may sequester Pax6 protein to inhibit its function as a transcriptional activator $^{15}$, TGF- $\beta$ s also function indirectly by inducing Wnt $2 b$. In turn, Wnt2b may repress Pax6 transcription directly through $\beta$-catenin/TCF. Although generally thought to be a transcriptional activator, new evidence suggests that this complex can also directly repress transcription ${ }^{21-22}$. In support of this idea, the exon 1 enhancer of Pax $6^{23}$ contains two conserved putative TCF-binding sites. The importance of $\beta$-catenin function in lens repression is also evident from studies in mouse, in which loss of $\beta$-catenin in the periocular ectoderm leads to the formation of small lentoids and rescues lens formation in Rx-deficient mice ${ }^{20,24}$. Thus, $\beta$-catenin has a critical role in preventing lens formation in inappropriate positions.

In addition, high levels of pSmad3 in the non-lens ectoderm may depend on a Wnt2b-mediated feed-forward loop: canonical Wnt signalling not only stabilizes $\beta$-catenin but also Smad3 through inhibition of GSK3 $\beta^{25-27}$, suggesting that sustained TGF- $\beta$ signalling is required to activate Smad3. Accordingly, pSmad3 stabilization directly correlates with high levels of canonical Wnt signalling induced by prolonged exposure to NCC-derived TGF- $\beta$ (compare midbrain and forebrain levels in Supplementary Fig. S2 and Fig. 3d,e). In contrast, Wnt signalling is absent in the future lens, partially because of the expression of Wnt antagonists ${ }^{20,28}$, and active GSK $3 \beta$ may mediate the degradation of both $\beta$-catenin and Smad 3 (refs 25-27). Consequently, Pax6 remains expressed and initiates downstream lens specification. Thus, Wnt-dependent Smad3 and $\beta$-catenin signalling may provide a robust switch between lens and non-lens fate. Interestingly, the same signals may also pattern the optic vesicle; specification of pigment epithelium depends on head mesenchyme-derived TGF- $\beta$ s, which in turn repress retinal fate ${ }^{29}$. Although $W n t 2 b$ is expressed in the pigment epithelium, its role in repressing retinal Pax6 has not been explored. Thus, Wnt and TGF- $\beta$ signalling may be used repeatedly to organize different components of the eye.

Our findings also highlight the fact that NCCs are crucial to ensure that central and peripheral parts of the eye become aligned in development: they prevent lens formation except next to the optic vesicle $^{4-6}$. The optic vesicle itself seems to have a dual role providing lens-promoting signals ${ }^{1,30-31}$ and functioning as a physical barrier to prevent apposition of NCCs and lens tissue. Like in chick, NCCs in humans are never in contact with the lens ${ }^{9}$, whereas in mouse they initially underlie the lens territory, but are subsequently displaced by the optic vesicle. This may explain why lens specification in mouse occurs only after optic vesicle contact ${ }^{30}$ and why lens formation fails in mouse mutants that lack the optic vesicle $e^{24,32-33}$.

In summary, NCCs emerge as key organizers of the vertebrate eye by contributing to the alignment of lens and retina and by patterning the optic vesicle. In addition, they have previously been implicated in coordinating the central (though not peripheral) projections from epibranchial placode-derived sensory ganglia ${ }^{34-35}$. It is therefore possible that one crucial function of NCCs lies in the integration of sensory structures, ensuring functional convergence of distinct embryonic components. The coemergence of both cell population ${ }^{36}$ may have offered a distinct advantage by enabling a diversity of complex sense organs to evolve in the vertebrate head.

\section{Methods}

Embryo manipulation. All experiments were performed in accordance with relevant guidelines and regulations of King's College London. Fertile hens' eggs (Henry Stewart) were incubated at $38^{\circ} \mathrm{C}$ to collect embryos between stages 8 and $10(\mathrm{HH}$ : Hamburger and Hamilton ${ }^{37}$ ). NCCs were ablated in ovo from $\mathrm{HH} 8$ embryos $^{4}$; using tungsten needles, the neural folds were removed bilaterally from posterior forebrain to anterior hindbrain levels. For in ovo electroporations ${ }^{38,}$ plasmid DNA $\left(2 \mu \mathrm{g} \mathrm{ml}^{-1}\right)$ was injected between the vitelline membrane and the surface ectoderm; four $50-\mathrm{ms}$ pulses of $7 \mathrm{~V}$ each were used at an interval of $1 \mathrm{~s}$ to transfect the surface ectoderm posterior to the lens or the PLE of HH8 embryos. The following constructs were used for electroporation: caß-catenin ${ }^{39}$, Smad7 (ref. 40) and caSmad3 (ref. 41).

Explant cultures. For explant cultures, PLE was dissected from HH8 embryos and freed from underlying mesoderm using $0.01 \%$ dispase and tungsten needles ${ }^{4}$. For NCC cocultures, PLEs were cultured with dorsal neural folds isolated from $\mathrm{HH} 8$ embryos at midbrain and posterior forebrain levels, which give rise to migrating NCCs during the culture period. To demonstrate TGF- $\beta$-dependent Wnt $2 b$ induction, migrating NCCs dorsal to the optic vesicle or lateral to the midbrain were dissected from $\mathrm{HH} 12-13$ embryos using tungsten needles and $0.01 \%$ dispase $^{4}$ All explants were placed on ice until use. Explants were cultured in 199 medium (Sigma), supplemented with $\mathrm{N}_{2}$ (Invitrogen), $\mathrm{NaHCO}_{3}\left(11 \mathrm{gl}^{-1}\right)$ and antibiotics. Collagen gels and culture media were supplemented with the following factors as required: $100-\mathrm{ng} \mathrm{ml}^{-1}$ Activin A (R\&D Systems), 500-ng ml ${ }^{-1}$ N-Frz-8 (R\&D Systems), 10- $\mu$ M SB431542/0.1\% dimethylsulphoxide (Tocris) or $0.1 \%$ dimethylsulphoxide only (controls).

In situ hybridization. Embryos and explants were processed for in situ hybridization $^{42}$ using DIG-labelled antisense probes for Six $1^{39}, \mathrm{Eya}^{43}, \mathrm{Pax}^{44}, \mathrm{~L}-\mathrm{Maf}^{45}$, $\delta$-crystallin ${ }^{4}, W n t 2 b$ (a gift from Susan Chapman), CRABP1 (a gift from Anthony Graham), TGF $\beta 1$, Activin $\beta A$, Activin $\beta B^{46}$ and Nodal ${ }^{47}$. After incubation with antiDIG antibodies (Roche) and washing, $4.5-\mu \mathrm{l}$ nitroblue tetrazolium (50 $\mathrm{mg} \mathrm{ml}^{-1}$ ) and 3.5- $\mu \mathrm{l}$ 5-bromo-4-chloro-3-indolyl phosphate $\left(50 \mathrm{mg} \mathrm{ml}^{-1}\right)$ per 1.5 - $\mathrm{ml}$ developing solution were used for colour development. Images were acquired using an AxioCam and Axiovision software; all explants were photographed at the same magnification $(\times 90)$ on an Olympus SX12.

Immunohistochemistry. Embryos were collected in PBS and fixed for $1 \mathrm{~h}$ at room temperature in 3.7\% formaldehyde/PBS, supplemented with 1:1000 Phosphatase Inhibitor Cocktail 2 (Sigma). Immunocytochemistry was performed on cryosections ${ }^{4}$, using HNK-1 antibody (1:10), antibodies against pSmad3 (Cell Signalling Technology; 1:100) and $\delta$-crystallin (a gift from Joram Piatigorsky; 1:2,500) and Alexa-coupled secondary antibodies (Invitrogen; 1:1000). All antibodies were diluted in blocking buffer (5\% goat serum, $0.1 \%$ Triton X-100 in PBS). Sections were photographed using a Leica LSM confocal microscope.

\section{References}

1. Chow, R. L. \& Lang, R. A. Early eye development in vertebrates. Annu. Rev. Cell Dev. Biol. 17, 255-296 (2001).

2. Spemann, H. Über Korrelationen in der Entwicklung des Auges. Verh. der Anat. Ges. 15, 61-79 (1901).

3. Saha, M. S., Spann, C. L. \& Grainger, R. M. Embryonic lens induction: more than meets the optic vesicle. Cell Differ. Dev. 28, 153-171 (1989).

4. Bailey, A. P., Bhattacharyya, S., Bronner-Fraser, M. \& Streit, A. Lens specification is the ground state of all sensory placodes, from which FGF promotes olfactory identity. Dev. Cell 11, 505-517 (2006).

5. Sullivan, C. H. et al. A re-examination of lens induction in chicken embryos: in vitro studies of early tissue interactions. Int. J. Dev. Biol. 48, 771-782 (2004).

6. von Woellwarth, C. Die rolle des neuralleistenmaterials und der temperatur bei der determination der augenlinse. Embryologia 6, 219-242 (1961).

7. Henry, J. J. \& Grainger, R. M. Inductive interactions in the spatial and temporal restriction of lens-forming potential in embryonic ectoderm of Xenopus laevis. Dev. Biol. 124, 200-214 (1987).

8. Le Douarin, N. M. \& Kalcheim, C. The neural Crest 2nd edn (Cambridge University Press, 1999).

9. O'Rahilly, R. \& Muller, F. The development of the neural crest in the human. J. Anat. 211, 335-351 (2007).

10. Grindley, J. C., Davidson, D. R. \& Hill, R. E. The role of Pax-6 in eye and nasal development. Development 121, 1433-1442 (1995).

11. Collinson, J. M., Hill, R. E. \& West, J. D. Different roles for Pax6 in the optic vesicle and facial epithelium mediate early morphogenesis of the murine eye. Development 127, 945-956 (2000).

12. Ashery-Padan, R., Marquardt, T., Zhou, X. \& Gruss, P. Pax6 activity in the lens primordium is required for lens formation and for correct placement of a single retina in the eye. Genes Dev. 14, 2701-2711 (2000). 
13. Aota, S. et al. Pax6 autoregulation mediated by direct interaction of Pax6 protein with the head surface ectoderm-specific enhancer of the mouse Pax6 gene. Dev. Biol. 257, 1-13 (2003).

14. Kleinjan, D. A., Seawright, A., Childs, A. J. \& van Heyningen, V. Conserved elements in Pax6 intron 7 involved in (auto)regulation and alternative transcription. Dev. Biol. 265, 462-477 (2004).

15. Grocott, T. et al. The MH1 domain of Smad3 interacts with Pax6 and represses autoregulation of the Pax6 P1 promoter. Nucleic Acids Res. 35, 890-901 (2007)

16. García-Campmany, L. \& Martí, E. The TGF $\beta$ intracellular effector Smad3 regulates neuronal differentiation and cell fate specification in the developing spinal cord. Development 134, 65-75 (2007).

17. Watabe, T. \& Miyazono, K. Roles of TGF- $\beta$ family signaling in stem cell renewal and differentiation. Cell Res. 19, 103-115 (2009).

18. Honda, A., Hirose, M. \& Ogura, A. Basic FGF and Activin/Nodal but not LIF signaling sustain undifferentiated status of rabbit embryonic stem cells. Exper. Cell Res. 315, 2033-2042 (2009).

19. Inman, G. J. et al. SB-431542 is a potent and specific inhibitor of transforming growth factor-beta superfamily type I activin receptor-like kinase (ALK) receptors ALK4, ALK5, and ALK7. Mol. Pharmacol. 62, 65-74 (2002).

20. Smith, A. N., Miller, L. A., Song, N., Taketo, M. M. \& Lang, R. A. The duality of beta-catenin function: a requirement in lens morphogenesis and signaling suppression of lens fate in periocular ectoderm. Dev. Biol. 285, 477-489 (2005).

21. Blauwkamp, T. A., Chang, M. V. \& Cadigan, K. M. Novel TCF-binding sites specify transcriptional repression by Wnt signalling. EMBO J. 27, 1436-1446 (2008).

22. Hoverter, N. P. \& Waterman, M. L. A Wnt-fall for gene regulation: repression. Sci. Signal. 1, pe43 (2008).

23. Zheng, J. B., Zhou, Y. H., Maity, T., Liao, W. S. \& Saunders, G. F. Activation of the human PAX6 gene through the exon 1 enhancer by transcription factors SEF and Sp1. Nucleic Acids Res. 29, 4070-4078 (2001).

24. Swindell, E. C. et al. Eye formation in the absence of retina. Dev. Biol. 322, 56-64 (2008).

25. Wu, D. \& Pan, W. GSK3: a multifaceted kinase in Wnt signaling. Trends Biochem. Sci. 35, 161-168 (2010).

26. Xu, C., Kim, N. G. \& Gumbiner, B. M. Regulation of protein stability by GSK3 mediated phosphorylation. Cell Cycle 8, 4032-4039 (2009).

27. Guo, X. et al. Axin and GSK3- control Smad3 protein stability and modulate TGF- signaling. Genes Dev. 22, 106-120 (2008).

28. Machon, O. et al. Lens morphogenesis is dependent on Pax6-mediated inhibition of the canonical Wnt/beta-catenin signaling in the lens surface ectoderm. Genesis 48, 86-95 (2010).

29. Fuhrmann, S., Levine, E. M. \& Reh, T. A. Extraocular mesenchyme patterns the optic vesicle during early eye development in the embryonic chick. Development 127, 4599-4609 (2000).

30. Furuta, Y. \& Hogan, B. L. BMP4 is essential for lens induction in the mouse embryo. Genes Dev. 12, 3764-3775 (1998).

31. Rajagopal, R. et al. The type I BMP receptors, Bmprla and Acvr1, activate multiple signaling pathways to regulate lens formation. Dev. Biol. 335, 305-316 (2009).

32. Mathers, P. H., Grinberg, A., Mahon, K. A. \& Jamrich, M. The Rx homeobox gene is essential for vertebrate eye development. Nature 387, 603-607 (1997).

33. Porter, F. D. et al. Lhx2, a LIM homeobox gene, is required for eye, forebrain, and definitive erythrocyte development. Development 124, 2935-2944 (1997).

34. Begbie, J. \& Graham, A. Integration between the epibranchial placodes and the hindbrain. Science 294, 595-598 (2001)

35. Coppola, E. et al. Epibranchial ganglia orchestrate the development of the cranial neurogenic crest. Proc. Natl Acad. Sci. USA 107, 2066-2071 (2010).

36. Northcutt, R. G. A reassessment of Goodrich's model of cranial nerve phylogeny. Acta. Anat. 148, 71-80 (1993).
37. Hamburger, V. \& Hamilton, H. L. A series of normal stages in the development of the chick embryo. J. Morph. 88, 49-92 (1951).

38. Bhattacharyya, S., Bailey, A. P., Bronner-Fraser, M. \& Streit, A. Segregation of lens and olfactory precursors from a common territory: cell sorting and reciprocity of Dlx5 and Pax6 expression. Dev. Biol. 271, 403-414 (2004).

39. Litsiou, A., Hanson, S. \& Streit, A. A balance of FGF, Wnt and BMP signalling positions the future placode territory in the head. Development 132, 4051-4062 (2005).

40. de Almeida, I. et al. Unexpected activities of Smad7 in Xenopus mesodermal and neural induction. Mech. Dev. 125, 421-431 (2008).

41. Garcia-Martinez, V. et al. State of commitment of prospective neural plate and prospective mesoderm in late gastrula/early neurula stages of avian embryos. Dev. Biol. 181, 102-115 (1997).

42. Streit, A. et al. Chordin regulates primitive streak development and the stability of induced neural cells, but is not sufficient for neural induction in the chick embryo. Development 125, 507-519 (1998).

43. Mishima, N. \& Tomarev, S. Chicken eyes absent 2 gene: isolation and expression pattern during development. Int. J. Dev. Biol. 42, 1109-1115 (1998).

44. Li, H. S., Yang, J. M., Jacobson, R. D., Pasko, D. \& Sundin, O. Pax-6 is first expressed in a region of ectoderm anterior to the early neural plate: implications for stepwise determination of the lens. Dev. Biol. 162, 181-194 (1994)

45. Ogino, H. \& Yasuda, K. Induction of lens differentiation by activation of a bZIP transcription factor, L-Maf. Science 280, 115-118 (1998).

46. Stern, C. D. et al. Activin and its receptors during gastrulation and the later phases of mesoderm development in the chick embryo. Dev. Biol. 172, 192-205 (1995).

47. Levin, M., Johnson, R. L., Stern, C. D., Kuehn, M. \& Tabin, C. A molecular pathway determining left-right asymmetry in chick embryogenesis. Cell $\mathbf{8 2}$, 803-814 (1995).

\section{Acknowledgments}

We thank Ewa Kolano for excellent technical assistance and Claudio Stern, Roberto Mayor and Ben Steventon for discussion and critical reading of the manuscript. This work was supported by grants from the BBSRC (D010659), the Wellcome Trust (081531) and Fight for Sight.

\section{Author contributions}

A.S. and T.G. conceived the project and designed the experiments; T.G. carried out most experiments, generated the figures and was involved in writing the manuscript. S.J. performed PLE/NCC coculture experiments in the presence of Wnt antagonists. A.P.B. performed some of the initial experiments and contributed to discussions A.S. contributed to PLE/NCC coculture experiments and wrote the manuscript.

\section{Additional information}

Supplementary Information accompanies this paper at http://www.nature.com/ naturecommunications

Competing financial interests: The authors declare no competing financial interests.

Reprints and permission information is available online at http://npg.nature.com/ reprintsandpermissions/

How to cite this article: Grocott, T. et al. Neural crest cells organize the eye via TGF- $\beta$ and canonical Wnt signalling. Nat. Commun. 2:265 doi: 10.1038/ncomms1269 (2011).

License: This work is licensed under a Creative Commons Attribution-NonCommercialShare Alike 3.0 Unported License. To view a copy of this license, visit http:// creativecommons.org/licenses/by-nc-sa/3.0/ 